\title{
FGFR4 c.1162G > A (p.Gly388Arg) Polymorphism Analysis in Turkish Patients with Retinoblastoma
}

\author{
Demet Akdeniz Odemis $\mathbb{D}^{1},{ }^{1}$ Seref Bugra Tuncer $\mathbb{D}^{1},{ }^{1}$ Arash Adamnejad Ghafour ${ }^{(\mathbb{D}}{ }^{1}$ \\ Khariga Jabbarli (iD), ${ }^{1}$ Yasemin Gider ${ }^{(D)},{ }^{1}$ Betul Celik (D), ${ }^{1}$ Gozde Kuru Turkcan ${ }^{(D)}{ }^{1}$ \\ Ozge Sukruoglu Erdogan $\mathbb{D}^{1},{ }^{1}$ Seda Kilic Erciyas $\mathbb{D}^{1},{ }^{1}$ Mukaddes Avsar $\mathbb{D}^{1},{ }^{1}$ Rejin Kebudi $\mathbb{D}^{2}$ \\ Sema Buyukkapu Bay $\mathbb{D}^{2}{ }^{2}$ Samuray Tuncer $\mathbb{D}^{3}{ }^{3}$ and Hulya Yazici $(\mathbb{D})^{1}$ \\ ${ }^{1}$ Istanbul University, Oncology Institute, Department of Basic Oncology, Division of Cancer Genetics, Istanbul, Turkey \\ ${ }^{2}$ Istanbul University, Oncology Institute, Division of Pediatric Hematology-Oncology, Istanbul, Turkey \\ ${ }^{3}$ Istanbul University, Istanbul Medical Faculty, Department of Ophthalmology, Istanbul, Turkey \\ Correspondence should be addressed to Hulya Yazici; hulyayazici67@gmail.com
}

Received 18 May 2020; Accepted 16 December 2020; Published 30 December 2020

Academic Editor: Federica Tomao

Copyright (c) 2020 Demet Akdeniz Odemis et al. This is an open access article distributed under the Creative Commons Attribution License, which permits unrestricted use, distribution, and reproduction in any medium, provided the original work is properly cited.

\begin{abstract}
Purpose. Various molecular variations are known to result in different gene variants in the FGFR4 gene, known for its oncogenic transformation activity. The goal of this study was to investigate the FGFR4 p.Gly388Arg variant that plays role in the progression of cancer and retinal growth and may be an effective candidate variant in the Turkish population in retinoblastoma patients with no RB1 gene mutation. Methods. Using the Sanger sequencing methods, the FGFR4 p.Gly388Arg variant was bidirectionally sequenced in 49 patients with non- $R B 1$ gene mutation in retinoblastoma patients and 13 healthy first-degree relatives and 146 individuals matched by sex and age in the control group. Results. In Turkish population-specific study, the FGFR4 p.Gly388Arg variant was found in 27 (55.1 percent) of 49 patients; mutation was found in 7 (53.8 percent) of these patients' 13 healthy relatives screened. When FGFR4 p.Gly388Arg mutation status is evaluated in terms of 146 healthy controls, in 70 (47.9 percent) individuals, mutation was observed. Our analysis showed that the FGFR4 p.Gly388Arg allele frequency, which according to different databases is seen as 30 percent in the general population, is 50 percent common in the Turkish population. Conclusions. In patients with advanced retinoblastoma who were diagnosed with retinoblastoma prior to 24 months, the FGFR4 p.Gly388Arg allele was found to be significantly higher. As a result, these results indicate that the polymorphism of FGFR4 p.Gly388Arg may play a role in both the development of tumors and the progression of aggressive tumors.
\end{abstract}

\section{Introduction}

Retinoblastoma is the most common primary intraocular malignancy in childhood and originates from primitive stem cells in the retina's nuclear layer [1]. Important genetic factors have been shown to play a role in the development of retinoblastoma [2]. According to existing literature, mutations in the retinoblastoma gene (RB1 (RefSeq NM-000321.2 and chromosome 13 coordinates in hg19)) [3] are believed to trigger the disease. However, how the disease progresses in patients who do not have an RB1 gene mutation scanned using the MLPA methods to assess minor insertions and deletions and sequencing of Sanger and large rearrangements is not clear. Many molecular changes that play an important role in the pathogenesis of tumors and the results of these changes have been seen in cancer patients in recent years. Growth factors, the oncogenic function of which was first identified, were found to play a role in the formation and development of tumors, activation of the cell cycle, formation of blood vessels, and escape from apoptotic control [4].

Today, the first isolated and most studied members of the family of fibroblast growth factor (FGF) are FGF-1 and FGF-2, which are considered to consist of at least 23 
structurally related polypeptic mitogens. In several pathophysiological processes, such as embryonic development, differentiation, neuronal survival, wound repair, and tumor formation, FGF family members play important roles in a wide range of tissues and cell types $[5,6]$. However, functions in angiogenesis, differentiation, and survival have been found in the FGF family [7]. With 5 distinct but very similar high affinity cell surface tyrosine kinase receptors (FGFR1, FGFR2, FGFR3, FGFR4, and FGFR5) [8], the FGF family has demonstrated its biological activities [8].

A member of the family of fibroblast growth factor (FGF) genes, the FGFR4 gene is also noted for its oncogenic transformation activity. Various molecular variations in the FGFR4 gene have been shown to lead to different variants of the gene. The FGFR4 variant (GRCh37 Chr5: 176520243, NM 002011.4: c.1162G > A p.Gly388Arg, rs351855 at the genotype level) is one of these variants, triggering the conversion of glycine amino acid to arginine amino acid at codon 388 [9]. FGFR4 p.Gly388Arg variant has been found to be correlated with development and disease prognosis of breast cancer [10], cervical cancer [11], hepatocellular carcinoma [12], thyroid cancer [13], gastric cancer [14], soft tissue tumors [15], lung cancer [9], colon cancer [16], prostate cancer [17], and head and neck tumors [18].

The FGFR4 gene is known to exert neuroprotective effects against the degeneration of retinal photoreceptors. The FGFR4 gene acts on the FGF-19 ligand, a molecule essential for photoreceptor ocular tissue formation and expressed by the embryonic retina. In mediating tumor growth and development, overexpression of FGFR4 and amplification of its target receptor FGF19 play a significant role. The abnormal signaling of FGF19-FGFR4 has been shown to influence the downstream signaling cascade involving particular tumorigenic events including cancer cell proliferation, resistance to apoptosis, and metastasis [19]. In this context, it can be thought that the variant of FGFR4 p.Gly388Arg in the FGFR4 gene, which is known to play a role in cancer progression and retinal development, may be a candidate mechanism and marker gene that triggers retinoblastoma formation. The effect of the FGFR4 p.Gly388Arg variant on retinoblastoma pathogenesis has been investigated in this planned study. This research indicates that the variant of FGFR4 p.Gly388Arg may have a population-specific polymorphism as a result of analysis performed in retinoblastoma patients and control groups after sequencing by the Sanger sequencing process.

\section{Materials and Methods}

2.1. Study Design and Patient Characteristics. The study was approved by the Local and Clinical Research Ethics Committee of Istanbul University (number of ethical approval: 2016-360), according to the tenets of the Declaration of Helsinki (JAMA 1997; 277:925-926). Written informed consent was obtained from all participants or parents of children under 18 years of age.
All patients consist of patients who applied to Istanbul University, Oncology Institute, Division of Pediatric Hematology-Oncology Istanbul University, Istanbul Medical Faculty, Department of Ophthalmology between 2011 and 2018. Although the study group was composed of a total of 208 individuals, all minor deletions and insertions were scanned predominantly by Sanger sequencing, large rearrangements were scanned with MLPA, and 49 individuals were selected for diagnosis of non- $R B 1$ gene mutation retinoblastoma. The mutation status of 13 healthy firstdegree relatives of the patients was also assessed in order to gain information on the family inheritance of the FGFR4 p.Gly388Arg variant. Differences between 146 age- and sexmatched healthy controls and patients with retinoblastoma were examined in order to determine the effect of this variant on retinoblastoma pathogenesis.

2.2. Features of the FGFR4 p.Gly388Arg Variant. In the NCBI reference sequence database (RefSeq) [20] and in algorithms such as Polymorphism Phenotyping (PolyPhen) [21] and SIFT [22], the FGFR4 p.Gly388Arg variant has been scanned. Data from the Catalog of Somatic Mutations in Cancer (COSMIC) [23], the ClinVar database [24], and the Online Mendelian Inheritance in Man (OMIM) catalogue [25] were used to test the relationship between the variant and the disease. The dbSNP [26], the Exome Aggregation Consortium (ExAC), the Genome Aggregation Database (gnomAD) [27], and the Ensemble 1000 Genome Project [28] were analyzed for information on the frequency and occurrence of variants in the population. The information gathered from the FGFR4 p.Gly388Arg variant data banks is shown in Table 1.

2.3. DNA Samples and Quality Checks. Peripheral blood samples were taken from all individuals involved in the analysis, and the Ficol (Sigma-Aldrich, Darmstadt, Germany) separation method was used for lymphocyte isolation. In compliance with the manufacturer's instructions, DNA isolation from these lymphocytes was carried out using the QIAamp DNA mini kit (Qiagen, 40724 Hilden, Germany). All DNA sample quality controls were carried out using the Qubit fluorimeter (ThermoFisher Science, Paisley PA4 9RF, UK) unit.

2.4. PCR Reaction and DNA Sequencing. For the sequencing of the FGFR4 p.Gly388Arg variant located in the $9^{\text {th }}$ Exon of the FGFR4 gene, two separate primers were designed in our study: forward (5'-TGACCAGTTTGTCTGTCTGTG-3 ') and reverse $\left(5^{\prime}\right.$-AGAACTGCAAAGTGGGAGAC-3'). Patient and control group samples were amplified by polymerase chain reaction using the designed primer pair (PCR). The PCR process was performed with a $50 \mu \mathrm{L}$ volume of $5 \mu \mathrm{l}$ Taq polymerase enzyme and a genomic DNA of $150 \mathrm{ng}$. Using the ABI Prism 3730 DNA Analyzer (Applied Biosystems, Foster City, CA) system, the PCR products 


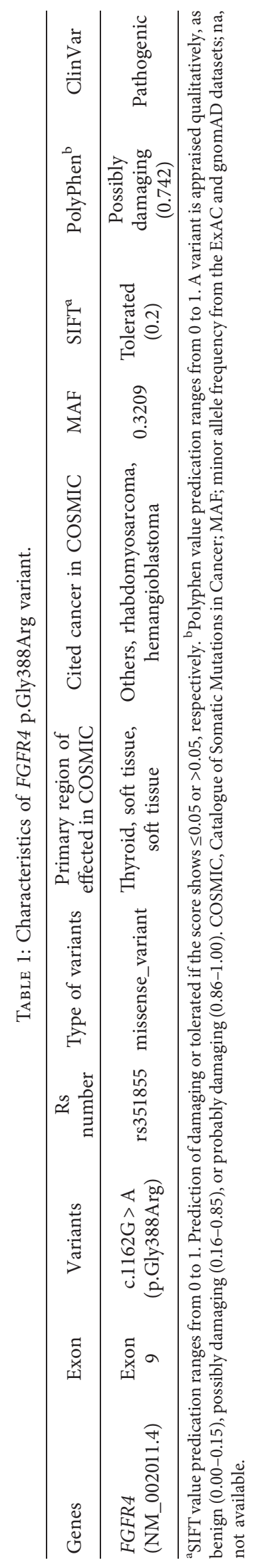


TABLE 2: Association of FGFR4 p.Gly388Arg variant with clinicopathologic variants.

\begin{tabular}{|c|c|c|c|c|c|}
\hline Clinicopathologic features & $\begin{array}{l}\text { Wild-type GG no. } \\
(\%)\end{array}$ & $\begin{array}{l}\text { Heterozygous GA no. } \\
(\%)\end{array}$ & $\begin{array}{l}\text { Homozygous AA no. } \\
(\%)\end{array}$ & $\begin{array}{l}\text { Total no. } \\
\quad(\%)\end{array}$ & $\begin{array}{l}\text { Significance, } \\
P \text { value }\end{array}$ \\
\hline \multicolumn{6}{|c|}{$\begin{array}{l}\text { Age (months), mean } 21 \pm 4, \text { range } \\
(1-216)\end{array}$} \\
\hline$<24$ months & $15(68.2 \%)$ & $11(50 \%)$ & $5(100 \%)$ & $31(63.3 \%)$ & \multirow{3}{*}{$0.04^{*}$} \\
\hline$\geq 24$ months & $7(31.8 \%)$ & $11(50 \%)$ & $0(0 \%)$ & $18(36.7 \%)$ & \\
\hline Total & 22 & 22 & 5 & 49 & \\
\hline \multicolumn{6}{|l|}{ Sex } \\
\hline Female & $15(68.2 \%)$ & $12(54.5 \%)$ & $1(20 \%)$ & $28(57.1 \%)$ & \multirow{3}{*}{0.137} \\
\hline Male & $7(31.8 \%)$ & $10(45.5 \%)$ & $4(80 \%)$ & $21(42.9 \%)$ & \\
\hline Total & 22 & 22 & 5 & 49 & \\
\hline \multicolumn{6}{|l|}{ Diagnosis } \\
\hline Unilateral & $18(81.8 \%)$ & $20(90.9 \%)$ & $5(100 \%)$ & $43(87.8 \%)$ & \multirow{3}{*}{0.444} \\
\hline Bilateral & $4(18.2 \%)$ & $2(9.1 \%)$ & $0(0 \%)$ & $6(12.2 \%)$ & \\
\hline Total & 22 & 22 & 5 & 49 & \\
\hline \multicolumn{6}{|l|}{ Leukocoria } \\
\hline Yes & $17(77.3 \%)$ & $16(72.7 \%)$ & $4(80 \%)$ & $37(75.5 \%)$ & \multirow{3}{*}{0.912} \\
\hline No & $5(22.7 \%)$ & $6(27.3 \%)$ & $1(20 \%)$ & $12(24.5 \%)$ & \\
\hline Total & 22 & 22 & 5 & 49 & \\
\hline \multicolumn{6}{|l|}{ Strabismus } \\
\hline Esodeviation & $10(45.5 \%)$ & $7(31.8 \%)$ & $2(40 \%)$ & $19(38.8 \%)$ & \multirow{4}{*}{0.929} \\
\hline Exodeviation & $4(18.1 \%)$ & $5(22.7 \%)$ & $1(20 \%)$ & $10(20.4 \%)$ & \\
\hline No & $8(36.4 \%)$ & $10(45.5 \%)$ & $2(40 \%)$ & $20(40.8 \%)$ & \\
\hline Total & 22 & 22 & 5 & 49 & \\
\hline \multicolumn{6}{|l|}{ Glaucoma } \\
\hline Yes & $5(22.7 \%)$ & $2(9.1 \%)$ & $0(0 \%)$ & $7(14.3 \%)$ & \multirow{3}{*}{0.201} \\
\hline No & $17(77.3 \%)$ & $20(90.9 \%)$ & $5(100 \%)$ & $42(85.7 \%)$ & \\
\hline Total & 22 & 22 & 5 & 49 & \\
\hline \multicolumn{6}{|l|}{ Stage } \\
\hline Early stage & $8(36.4 \%)$ & $3(13.6 \%)$ & $0(0 \%)$ & $11(22.4 \%)$ & \multirow{3}{*}{$0.031^{*}$} \\
\hline Late stage & $14(63.6 \%)$ & $19(86.4 \%)$ & $5(100 \%)$ & $38(77.6 \%)$ & \\
\hline Total & 22 & 22 & 5 & 49 & \\
\hline \multicolumn{6}{|l|}{ Treatment } \\
\hline \multicolumn{6}{|l|}{ IAC } \\
\hline Yes & $10(45.5 \%)$ & $12(54.5 \%)$ & $4(80 \%)$ & $26(53.1 \%)$ & \multirow{3}{*}{0.370} \\
\hline No & $12(54.5 \%)$ & $10(45.5 \%)$ & $1(20 \%)$ & $23(46.9 \%)$ & \\
\hline Total & 22 & 22 & 5 & 49 & \\
\hline \multicolumn{6}{|l|}{$\mathrm{CT}$} \\
\hline Yes & $10(45.5 \%)$ & $8(36.4 \%)$ & $1(20 \%)$ & $19(38.8 \%)$ & \multirow{3}{*}{0.546} \\
\hline No & $12(54.5 \%)$ & $14(63.6 \%)$ & $4(80 \%)$ & $30(61.2 \%)$ & \\
\hline Total & 22 & 22 & 5 & 49 & \\
\hline \multicolumn{6}{|l|}{ RT } \\
\hline Yes & $2(9.1 \%)$ & $0(0 \%)$ & $0(0 \%)$ & $2(4.1 \%)$ & \multirow{3}{*}{0.278} \\
\hline No & $20(90.9 \%)$ & $22(100 \%)$ & $5(100 \%)$ & 47 (95.9\%) & \\
\hline Total & 22 & 22 & 5 & 49 & \\
\hline Surgery (enucleation) & & & & & \\
\hline Yes & $11(50 \%)$ & $9(40.9 \%)$ & $0(0 \%)$ & $20(40.8 \%)$ & \\
\hline No & $11(50 \%)$ & $13(59.1 \%)$ & $5(100 \%)$ & $29(59.2 \%)$ & 0.05 \\
\hline Total & 22 & 22 & 5 & 49 & \\
\hline LOT & & & & & \\
\hline Yes & $3(13.6 \%)$ & $4(18.2 \%)$ & $0(0 \%)$ & $7(14.3 \%)$ & \\
\hline No & $19(86.4 \%)$ & $18(81.8 \%)$ & $5(100 \%)$ & $42(85.7 \%)$ & 0.573 \\
\hline Total & 22 & 22 & 5 & 49 & \\
\hline
\end{tabular}


obtained were bidirectionally sequenced and sequencing data were analyzed using SnapGene Viewer 4.0.5 Software.

2.5. Statistical Analysis. Our main objective was to obtain information on the family inheritance of the variant FGFR4 p.Gly388Arg and to assess its effect on the pathogenesis of retinoblastoma. In order to estimate the distribution of the FGFR4 p.Gly388Arg variant in the population and to compare the genotype variations between the patient and control groups, a chi-square $\left(X^{2}\right)$ test was carried out using categorical variables. In addition, to equate the clinicopathological and epidemiological parameters with the FGFR4 variant p.Gly388Arg, the $X^{2}$ test was used. A $p$ value of $<0.05$ was found statistically relevant. All statistical analyses were performed using Windows (SPSS, Chicago, IL, USA) version 21.0 SPSS software.

\section{Results}

Scope of work, a total of 8 criteria, including stratification analysis, were evaluated in terms of clinicopathological characteristics: month of diagnosis, sex, diagnosis, leukocoria, strabismus, glaucoma, and treatment. Table 2 displays all the clinicopathological characteristics in the cases. The diagnostic age (months) of the patients ranged from 1 to 216 months, while the mean age was 21 months. Although 31 (63.3 percent) of 49 patients with retinoblastoma were diagnosed prior to 24 months, 18 (36.7 percent) were diagnosed after 24 months. 16 (59.3\%) of patients diagnosed before 2 years of age and 11 (40.7\%) of patients diagnosed after 2 years of age were positive for the p.Gly388Arg FGFR4 mutation $\left(p=0.04^{*}\right)$. In terms of the FGFR4 p.Gly388Arg mutation, 18 (51.4 percent) of 35 (56.5 percent) female patients and 16 (59.3 percent) of 27 (43.5 percent) male patients were considered positive. In 25 (58.1\%) of 43 $(87.7 \%)$ patients with unilateral retinoblastoma, the FGFR4 p.Gly388Arg mutation was detected; in 2 (33.3\%) of 6 (12.3\%) patients with bilateral retinoblastoma, the FGFR4 p.Gly388Arg mutation was identified. In 25 (58.1\%) of 43 (87.7\%) patients with unilateral retinoblastoma, the FGFR4 p.Gly388Arg mutation was detected; in $2(33.3 \%)$ of 6 (12.3\%) patients with bilateral retinoblastoma, the FGFR4 p.Gly388Arg mutation was identified. 29 (59.2\%) patients with strabismus disease applied to the doctor, $19(65.5 \%)$ of these patients had esodeviation, and 10 (34.5 percent) had exodeviation. In 15 (51.7 percent) of these strabismus patients, the FGFR4 p.Gly388Arg mutation was found to be positive. In 7 (14.3 percent) of the patients at the time of diagnosis, glaucoma was seen, but only 2 (28.6 percent) of these patients had mutations.

Although 38 (77.6\%) of 49 patients with retinoblastoma were diagnosed with advanced stage (group C, group D, or group E) retinoblastoma, 11 (22.4\%) were diagnosed with early-stage retinoblastoma, when the clinical stages of the patients were assessed (group A and group B). Although 24 $(88.8 \%)$ of the 27 FGFR4 p.Gly388Arg mutation patients were in the advanced stage, $3(11.1 \%)$ were diagnosed early. A statistically relevant association between early stage and advanced stage patients $(p=0.031)$ was found in terms of FGFR4 p.Gly388Arg mutation when the clinical stage and FGFR4 p.Gly388Arg mutation status were evaluated. 26 (53.1 percent) had intra-arterial chemotherapy (IAC), 19 (38.8 percent) had systemic chemotherapy (CT) for chemoreduction, 2 (4.1 percent) had regional radiotherapy (RT), 7 (14.3 percent) had local ophthalmic treatment (LOT) (cryotherapy, thermotherapy, and laser therapy), and 20 (40.8 percent) were determined to have undergone treatment (LOT) (cryotherapy, thermotherapy, and laser therapy) and 20 (40.8 percent) enucleation of eye. There was no statistically relevant association between the p.Gly388Arg mutation in FGFR4 and the patients' treatment choices $(p \geq 0.05)$.

It was found that 3 (6.1 percent) patients had a history of first-degree-associated retinoblastoma when evaluating the association of the FGFR4 p.Gly388Arg variant with family history. These were all patients with heterozygous GA alleles; c. $[1162 \mathrm{G}>\mathrm{G}]$; the $[1162 \mathrm{G}>\mathrm{A}]$ mutation was introduced. In this study, in patients with a family history of retinoblastoma, the FGFR4 p.Gly388Arg mutation rate was recognized as "almost significant" (e.g., $\left.p=0.08^{* *}\right) .36$ (73.5 percent) patients have been identified when the presence of tumors other than retinoblastoma in the patient family is investigated. Of these patients, 20 (55.5 percent) were found to be positive for the p.Gly388Arg FGFR4 mutation and 16 (45.5 percent) were negative $(p=0.94)$. It was reported that the family members of $31(63.3 \%)$ patients were included in each of these occupational groups when the next three generations are studied in terms of risk evaluation of occupational groups (machinists, welders, metal industry, textile, building, and military). In 19 (61.3\%) of these patients, the FGFR4 p.Gly388Arg variant was identified ( $p=0.07$ is nearly significant). Table 3 indicates the association of the FGFR4 p.Gly388Arg variant with family history.

The Wild-type GG allele rate was 104 (50\%), heterozygous GA allele rate 84 (40.4\%), and homozygous AA allele rate 20 (9.6\%) among 208 samples. In 27 (55.1 percent) of 49 patients, the FGFR4 p.Gly388Arg variant was found positive; 22 (81.5 percent) carried the heterozygous GA allele and 5 (18.5 percent) carried the homozygous AA allele. 5 (71.4 percent) of the 7 individuals were found to have the heterozygous GA allele and 2 (28.6 percent) the homozygous $\mathrm{AA}$ allele as a result of the screening of 13 healthy relatives of these patients. When the mutation status of FGFR4 p.Gly388Arg is tested in terms of 146 healthy controls, mutations were observed in 70 individuals, 57 (81.4\%) were heterozygous GA allele, and 13 (18.6\%) were homozygous AA allele. In terms of the FGFR4 p.Gly388Arg mutation $(p=0.86)$, there was no statistically significant difference between the patient and control groups. Table 4 and Figure 1 display the distribution of the FGFR4 p.Gly388Arg (c.1162G > A) variant in patients, relatives, and control groups.

\section{Discussion}

Cancer is a hereditary disorder that occurs in several genes as a result of mutations that play role in the division, 
TABLE 3: Association of FGFR4 p.Gly388Arg variant with family history.

\begin{tabular}{|c|c|c|c|c|c|}
\hline Family history & Wild-type GG no. (\%) & Heterozygous GA no. (\%) & Homozygous AA no. (\%) & Total no. (\%) & Significance, $p$ value \\
\hline \multicolumn{6}{|l|}{$\mathrm{RB}$ in family } \\
\hline Yes & $0(0 \%)$ & $3(13.6 \%)$ & $0(0 \%)$ & $3(6.1 \%)$ & \\
\hline No & $22(100 \%)$ & $19(86.4 \%)$ & $5(100 \%)$ & $46(93.9 \%)$ & 0.08 \\
\hline Total & 22 & 22 & 5 & 49 & \\
\hline \multicolumn{6}{|l|}{ OT in family } \\
\hline Yes & $16(72.7 \%)$ & $16(72.7 \%)$ & $4(80 \%)$ & $36(73.5 \%)$ & \\
\hline No & $6(27.3 \%)$ & $6(27.3 \%)$ & $1(20 \%)$ & $13(26.5 \%)$ & 0.94 \\
\hline Total & 22 & 22 & 5 & 49 & \\
\hline \multicolumn{6}{|l|}{ RAOG in family } \\
\hline Yes & $12(54.5 \%)$ & $14(63.6 \%)$ & $5(100 \%)$ & $31(63.3 \%)$ & \\
\hline No & $10(45.5 \%)$ & $8(36.4 \%)$ & $0(0 \%)$ & $18(36.7 \%)$ & 0.07 \\
\hline Total & 22 & 22 & 5 & 49 & \\
\hline
\end{tabular}

Wild-type GG allele; c.[1162G > G]; [1162G> G], heterozygous GA allele; c. [1162G > G]; [1162G>A], homozygous AA; c. [1162G>G]; [1162A > A]. RB, retinoblastoma; OT, other tumors (brain tumor, breast cancer, lung cancer, thyroid cancer, and prostate cancer); RAOG, risk assessment of occupational groups.

TABLE 4: Distribution of FGFR4 p.Gly388Arg (c.1162G > A) variant in patients, relatives, and control group.

\begin{tabular}{lccccc}
\hline FGFR4 p.Gly388Arg (c.1162G > A) & Patients no. (\%) & Relatives no. (\%) & Control no. (\%) & Total no. (\%) & Significance, $p$ value \\
\hline Wild-type GG & $22(21.2 \%)$ & $6(5.7 \%)$ & $76(73.1 \%)$ & $104(50 \%)$ \\
Heterozygous GA & $22(26.2 \%)$ & $5(6 \%)$ & $57(67.8 \%)$ & $84(40.4 \%)$ & $20(9.6 \%)$ \\
Homozygous AA & $5(25 \%)$ & $2(10 \%)$ & $13(65 \%)$ & 208 & 0.86 \\
Total & 49 & 13 & 146 & 208 \\
\hline
\end{tabular}

Wild-type GG allele; c. [1162G > G]; [1162G > G], heterozygous GA allele; c. [1162G>G]; [1162G>A], homozygous AA; c. [1162G>G]; [1162A>A].

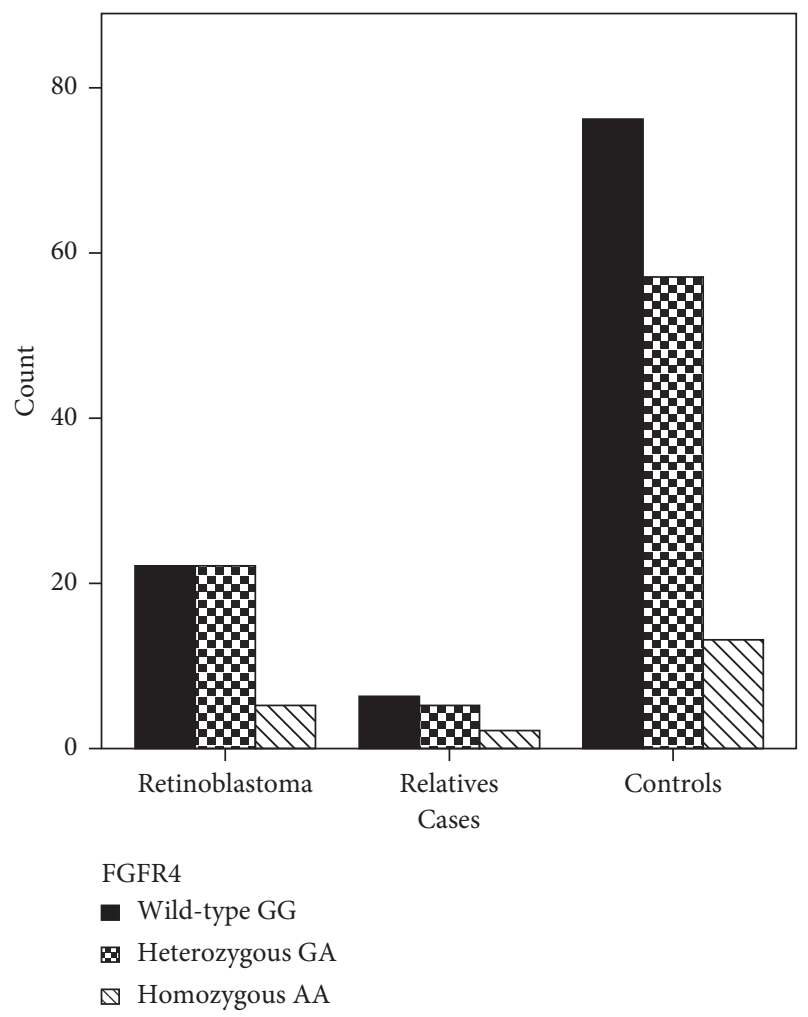

FIGURE 1: The distribution of FGFR4 p.Gly388Arg (c.1162G > A) variant in retinoblastoma patients, relatives, and control group.

proliferation, and death of cells. Retinoblastoma develops in the eye and is the most prevalent childhood intraocular malignancy. Important genetic factors are believed to play a role in the development of retinoblastoma. It is understood, according to current literature, that the disease is caused by mutations in a gene called the retinoblastoma. It is not clear, however, how the disorder progresses in patients who are found to be negative as a result of $R B 1$ gene mutation scans.

The FGFR4 gene is known to exert neuroprotective effects against the degeneration of retinal photoreceptors. In photoreceptors, FGFR4 expression affects a particular ligand, FGF-19. FGF-19 is a molecule that is essential and expressed by the embryonic retina for the development of ocular tissue. There are also several studies in the literature that examine the possible role of FGF-19 in the mechanism of photoreceptors. Siffroi-Fernandez et al., in 2008, stressed the neuroprotective effects of FGF-19 on mammalian photoreceptors [29]. Lang et al., in 2018, showed that overexpression of FGFR4 and amplification of its target FGF19 receptor played an important role in mediating tumor growth and progression [19]. Villalonga et al, in their 2018 report, emphasized that the variant FGFR4 p.Gly388Arg initiated tumor progression by stimulating the expression of $\mathrm{N}$-cadherin protein in lung cancer patients [9]. The variant FGFR4 p.Gly388Arg has been shown to penetrate the STAT3 molecule into the cell membrane and impact cell surface molecules, thereby accelerating the progression of cancer and becoming an important risk factor for the progression of the disease [30]. Bange et al. observed in their study that the FGFR4 p.Gly388Arg variant was associated with cancer progression and cell motility [31]. A candidate mechanism and marker gene that activates the formation of retinoblastoma in this context may be considered to be the FGFR4 p.Gly388Arg variant in the FGFR4 
gene, which is known to be involved in the development of cancer and retinal growth.

In this study, the effect of the FGFR4 p.Gly388Arg variant on 49 nonmutated patients with retinoblastoma of the $R B 1$ gene, their 13 healthy relatives and 146 age- and gender-matched control groups, and the FGFR4 $\mathrm{p}$ Gly388Arg variant on patients with retinoblastoma and control groups used the bidirectional sequencing Sanger Sequencing process. As a result, we have investigated whether there could be a mechanism that initiates retinoblastoma oncogenesis.

Our first goal in this study was to establish if the variant FGFR4 p.Gly388Arg may be another primary mechanism in the formation of retinoblastoma other than the $R B 1$ gene. In the research, no statistically significant difference in mutation in the FGFR4 p.Gly388Arg variant was identified between the patient and control groups. It was also assumed that the FGFR4 p.Gly388Arg variant was a populationspecific polymorphism.

The most significant finding of this study is the explanation of the effect of a polymorphism in patients who have no $R B 1$ mutation but are diagnosed with $\mathrm{RB}$, triggering the conversion of the glycine amino acid to the amino acid of arginine at the 388 codon of the human FGFR 4 gene. When the FGFR4 p.Gly388Arg variant minor allele frequency is screened in the ExAC and Genome Aggregation Database (gnomAD) datasets, it appears to be a known polymorphism in the general population with a MAF ratio of 0.3209 . In our Turkish population-specific research, however, the FGFR4 p.Gly388Arg variant was found to be positive in 27 (55.1 percent) of 49 patients; mutation was found in 7 (53.8 percent) of these patients' 13 healthy relatives screened. When FGFR4 p.Gly388Arg mutation status is evaluated in terms of 146 healthy controls, in 70 (47.9 percent) individuals, mutation was observed. As a result, in 104 (50 percent) of 208 individuals screened in total, the FGFR4 p.Gly388Arg mutation was detected. In view of all these findings, our analysis has shown that the frequency of FGFR4 p.Gly388Arg allele, which according to various databases is seen as 30 percent in the general population, is prevalent in the Turkish population at a rate of 50 percent.

Nonetheless, our statistical study clearly showed that, in advanced stage patients diagnosed with retinoblastoma prior to 24 months, the FGFR4 p.Gly388Arg allele was significantly higher. As a result, these findings suggest that the FGFR4 p.Gly388Arg allele may play a role in both aggressive tumor progression and tumor formation. Furthermore, taking into account the status of the FGFR4 p.Gly388Arg allele, this polymporphism can be considered to be a novel alternative for predicting clinical development and assessing the stage of disease in patients with advanced stage retinoblastoma. Studies on a wider community of patients with longer follow-up data will help to test this theory. The findings to be obtained from future studies are expected to contribute to the literature on the pathogenesis, etiology, and genetic origin of retinoblastoma and to direct the next generation in terms of early detection and options for treatment. In addition, genetic testing in the prenatal and preimplantation phases in new family pregnancies may be conducted to predict the disease until the baby is born, or the gene-borne disease in the family can be fully removed by in vitro fertilization (ivf).

\section{Data Availability}

The data used to support the findings of this study are restricted by the Local and Clinical Research Ethics Committee of Istanbul University in order to protect patient privacy.

\section{Ethical Approval}

This study complies with ethical standards. The conformity approval of the Ethics Board was granted to conduct the study in Istanbul University, Oncology Institute, Department of Basic Oncology with the date 18.05.2016 and no. 360 .

\section{Consent}

All patients were informed about the study, and consents were granted in the scope of the cancer genetics polyclinic. Individual consent was signed by each individual.

\section{Conflicts of Interest}

The authors declare that they have no conflicts of interest.

\section{Acknowledgments}

This work was supported by Scientific Research Projects Coordination Unit of Istanbul University (Project number: BYP-2018-27990).

\section{References}

[1] M. V. Ortiz and I. J. Dunkel, "Retinoblastoma," Journal of Child Neurology, vol. 31, no. 2, pp. 227-236, 2016.

[2] D. Qi, M. Wang, and F. Yu, "Knockdown of lncRNA-H19 inhibits cell viability, migration and invasion while promotes apoptosis via microRNA-143/RUNX2 axis in retinoblastoma," Biomedicine \& Pharmacotherapy, vol. 109, pp. 798805, 2019.

[3] M. Jagadeesan, V. Khetan, and A. Mallipatna, "Genetic perspective of retinoblastoma: from present to future," Indian Journal of Ophthalmology, vol. 64, no. 5, pp. 332-336, 2016.

[4] S. Siffroi-Fernandez, "Acidic fibroblast growth factor (FGF-1) and FGF receptor 1 signaling in human Y79 retinoblastoma," Archives of Ophthalmology, vol. 123, no. 3, pp. 368-376, 2005.

[5] J. Kandel, E. Bossy-Wetzel, F. Radvanyi, M. Klagsbrun, J. Folkman, and D. Hanahan, "Neovascularization is associated with a switch to the export of bFGF in the multistep development of fibrosarcoma," Cell, vol. 66, no. 6, pp. 1095-1104, 1991.

[6] W. L. McKeehan, F. Wang, and M. Kan, "The heparan sulfatefibroblast growth factor family: diversity of structure and function," Progress in Nucleic Acid Research and Molecular Biology, vol. 59, pp. 135-176, 1998.

[7] V. Fontaine, N. Kinkl, J. Sahel, H. Dreyfus, and D. Hicks, "Survival of purified rat photoreceptors in vitro is stimulated directly by fibroblast growth factor-2," The Journal of Neuroscience, vol. 18, no. 23, pp. 9662-9672, 1998. 
[8] C. J. Powers, S. W. McLeskey, and A. Wellstein, "Fibroblast growth factors, their receptors and signaling," EndocrineRelated Cancer, vol. 7, no. 3, pp. 165-197, 2000.

[9] A. Quintanal-Villalonga, "The FGFR4-388arg variant promotes lung cancer progression by $\mathrm{N}$-cadherin induction," Scientific Reports, vol. 8, no. 1, p. 2394, 2018.

[10] X. Zhao, F. Xu, N. P. Dominguez et al., "FGFR4 provides the conduit to facilitate FGF19 signaling in breast cancer progression," Molecular Carcinogenesis, vol. 57, no. 11, pp. 1616-1625, 2018.

[11] L. Guo, A. Zhao, R. Zhang, Y. Xie, and X. Xu, "Study on the association between the fibroblast growth factor receptor 4 expression and high-risk human papillomavirus infection in cervical cancer and precancerous lesions," Gynecologic and Obstetric Investigation, vol. 83, no. 6, pp. 608-614, 2018.

[12] G. Cui, R. C. Martin, H. Jin et al., "Up-regulation of FGF15/19 signaling promotes hepatocellular carcinoma in the background of fatty liver," Journal of Experimental \& Clinical Cancer Research, vol. 37, no. 1, p. 136, 2018.

[13] E. Motylewska, T. Stępień, M. Borkowska et al., "Alteration in the serum concentrations of FGF19, FGFR4 and $\beta$ Klotho in patients with thyroid cancer," Cytokine, vol. 105, pp. 32-36, 2018.

[14] T. Yu, L.-N. Wang, W. Li et al., "Downregulation of miR-491$5 p$ promotes gastric cancer metastasis by regulating SNAIL and FGFR4," Cancer Science, vol. 109, no. 5, pp. 1393-1403, 2018.

[15] T. McKinnon, R. Venier, M. Yohe et al., "Functional screening of FGFR4-driven tumorigenesis identifies PI3K/mTOR inhibition as a therapeutic strategy in rhabdomyosarcoma," Oncogene, vol. 37, no. 20, pp. 2630-2644, 2018.

[16] D. Wang, J. Zhang, Z. Li et al., "Upregulation of fibroblast growth factor 19 is associated with the initiation of colorectal adenoma," Digestive Diseases, vol. 37, no. 3, pp. 214-225, 2018.

[17] S. E. A. Burnell, S. S. Harty, S. Howarth et al., "STEAP2 knockdown reduces the invasive potential of prostate cancer cells," Scientific Reports, vol. 8, no. 1, p. 6252, 2018.

[18] L. Gao, L. Lang, X. Zhao, C. Shay, A. Y. Shull, and Y. Teng, "FGF19 amplification reveals an oncogenic dependency upon autocrine FGF19/FGFR4 signaling in head and neck squamous cell carcinoma," Oncogene, vol. 38, no. 13, pp. 2394-2404, 2018.

[19] L. Lang, A. Y. Shull, and Y. Teng, "Interrupting the FGF19FGFR4 axis to therapeutically disrupt cancer progression," Current Cancer Drug Targets, vol. 19, no. 1, pp. 17-25, 2018.

[20] N. A. O’Leary, M. W. Wright, J. R. Brister et al., "Reference sequence (RefSeq) database at NCBI: current status, taxonomic expansion, and functional annotation," Nucleic Acids Research, vol. 44, no. D1, pp. D733-D745, 2016.

[21] I. A. Adzhubei, S. Schmidt, L. Peshkin et al., "A method and server for predicting damaging missense mutations," Nature Methods, vol. 7, no. 4, pp. 248-249, 2010.

[22] P. Kumar, S. Henikoff, and P. C. Ng, "Predicting the effects of coding non-synonymous variants on protein function using the SIFT algorithm," Nature Protocols, vol. 4, no. 7, pp. 1073-1081, 2009.

[23] S. A. Forbes, D. Beare, H. Boutselakis et al., "COSMIC: somatic cancer genetics at high-resolution," Nucleic Acids Research, vol. 45, no. D1, pp. D777-D783, 2017.

[24] M. J. Landrum, J. M. Lee, M. Benson et al., "ClinVar: improving access to variant interpretations and supporting evidence," Nucleic Acids Research, vol. 46, no. D1, pp. D1062-D1067, 2018.
[25] V. A. McKusick, "Mendelian inheritance in man and its online version, OMIM," The American Journal of Human Genetics, vol. 80, no. 4, pp. 588-604, 2007.

[26] S. T. Sherry, "dbSNP: the NCBI database of genetic variation," Nucleic Acids Research, vol. 29, no. 1, pp. 308-311, 2001.

[27] M. Lek, K. J Karczewski, E. V Minikel et al., "Analysis of protein-coding genetic variation in 60,706 humans," Nature, vol. 536, no. 7616, pp. 285-291, 2016.

[28] Genomes Project Consortium, A. Auton, L. D. Brooks et al., "A global reference for human genetic variation," Nature, vol. 526, no. 7571, pp. 68-74, 2015.

[29] S. Siffroi-Fernandez, M.-P. Felder-Schmittbuhl, H. Khanna, A. Swaroop, and D. Hicks, "FGF19 exhibits neuroprotective effects on adult mammalian photoreceptors in vitro," Investigative Opthalmology \& Visual Science, vol. 49, no. 4, pp. 1696-1704, 2008.

[30] V. K. Ulaganathan, B. Sperl, U. R. Rapp, and A. Ullrich, "Germline variant FGFR4 p.G388R exposes a membraneproximal STAT3 binding site,” Nature, vol. 528, no. 7583, pp. 570-574, 2015.

[31] J. Bange, D Prechtl, Y Cheburkin et al., "Cancer progression and tumor cell motility are associated with the FGFR4 Arg(388) allele," Cancer Research, vol. 62, no. 3, pp. 840-847, 2002. 